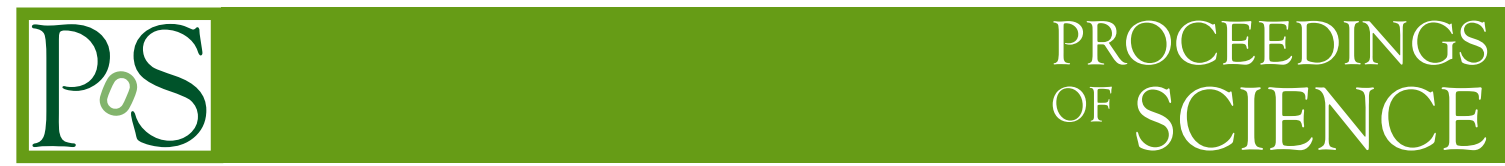

\title{
CP violation in the baryon sector
}

\author{
Eluned Smith* \\ RWTH Aachen \\ E-mail: eluned.anne.smith@cern.ch

\section{On behalf of the LHCb collaboration}

The study of $\mathrm{CP}$ violation in the baryon sector is still a relatively new field and offers the possibility to make many $\mathrm{CP}$ measurements which could complement those performed in the meson sector. This is especially true of late given the large number of baryons currently being produced at the LHC. Such measurements could help further over-constrain the CKM unitary triangle, as well as furthering our understand of baryongenesis. These proceedings will give an overview of the current state of the search for $\mathrm{CP}$ violation in the baryon sector.

The 15th International Conference on Flavor Physics and CP Violation

5-9 June 2017

Prague, Czech Republic

${ }^{*}$ Speaker. 


\section{Why look for CP violation in the baryon sector?}

The presence of CP violation (CPV) in the meson sector was first discovered in 1963 by Cronin and Fitch [1] and since then has been observed and well measured in $B^{0}, B^{+}$and $B_{s}^{0}$ decays. However, despite the increasing precision on both experimental results and the Standard Model (SM) predictions, no significant deviations from the SM have been seen in the meson sector.

The baryon sector offers the possibility to significantly increase the number of measurements made on CKM triangle parameters, as well as helping to further our understanding of baryongenesis.

\section{Experimental challenges when searching for $\mathrm{CP}$ violation in the baryon sector}

There are various experimental parameters which could be sensitive to $\mathrm{CP}$ violation, the most straight forward being the asymmetry, $A_{C P}$, defined as $\frac{N-\bar{N}}{N+\bar{N}}$, where $N(\bar{N})$ refers to the number of decays (charge conjugate decays) observed.

However, in order to extract the true value of $A_{C P}$ the production and reconstruction asymmetries must also be well understood. Unfortunately, measuring the production asymmetry is non-trivial at hadron colliders, and therefore, where possible, it is often more desirable to use experimental parameters which are not sensitive to production asymmetries.

Of relevance to the analyses outlined in these proceedings are the experimental parameters $\Delta A_{C P}$ and so-called triple products. The quantity $\Delta A_{C P}$ is defined as the difference in $A_{C P}$ between two decays that have the same mother, and ideally the same final states. Thus, by taking the difference in $A_{C P}$ between such two decays, the unknown production asymmetries cancel.

Alternatively, one can also construct observable which are CP-odd and, by construction, not sensitive to production asymmetries. There are various ways to form such CP-odd observables and one method is via asymmetries under the $\hat{T}$ and $P$ operators, where here $\hat{T}$ is an unitary operator which reverses both the momentum and spin three-vectors [2,3]. The precise formation of these CP-odd observables will be outlined in section 5 .

\section{Search for CPV in $\Lambda_{b}^{0} \rightarrow p \pi^{-}\left(K^{-}\right)$decays at $\mathrm{CDF}$}

The decays $\Lambda_{b}^{0} \rightarrow p \pi^{-}\left(K^{-}\right)$are the baryonic equivalent of the decays $B^{0} \rightarrow K^{+}\left(\pi^{+}\right) \pi^{-}$, as demonstrated by the Feynman diagrams in Fig. 1. This decay occurs via either a tree level process involving the CKM elements $V_{u b} V_{u d}^{*}$, or via a loop involving the CKM elements $V_{t b} V_{t d}^{*}$. Given that $\mathrm{CPV}$ can arise from the interference of two amplitudes with relative phases that differ between particle and antiparticle decays, and the large relative phase between $V_{u b} V_{u d}^{*}$ and $V_{t b} V_{t d}^{*}$, these decays could be particularly sensitive to CPV effects.

In addition, the theoretical predictions for the amount of CPV in this decay are very precise, with a relative error of $\sim 3 \%$ [4]. In Ref. [4] the value for CPV in $\Lambda_{b}^{0} \rightarrow p \pi^{-}\left(K^{-}\right)$decays is predicted to be around 5\%. A search for CPV in $\Lambda_{b}^{0} \rightarrow p \pi^{-}\left(K^{-}\right)$decays was performed by CDF in 2014 and was found to be consistent with both the SM predictions and zero [5], with uncertainties 


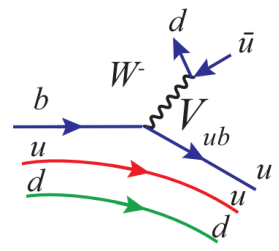

$\Lambda_{b}^{0} \rightarrow p \pi^{-}$

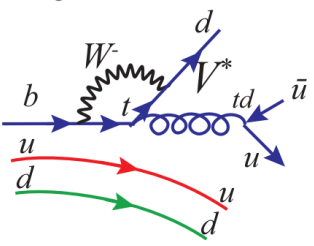

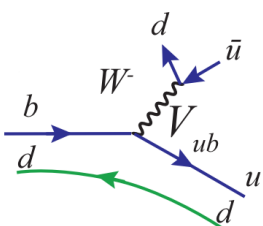

$B_{d}^{0} \rightarrow \pi^{+} \pi^{-}$

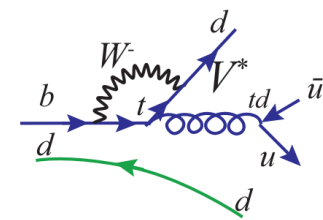

Figure 1: Comparison of Feynman diagrams for $\Lambda_{b}^{0} \rightarrow p \pi^{-}$decays (left) and $B^{0} \rightarrow K^{+} \pi^{-}$decays (right).

being dominated by statistics:

$$
\begin{aligned}
& A_{C P}\left(\Lambda_{b}^{0} \rightarrow p \pi^{-}\right)=+0.06 \pm 0.07(\text { stat }) \pm 0.03(\text { syst }) \\
& A_{C P}\left(\Lambda_{b}^{0} \rightarrow p K^{-}\right)=+0.10 \pm 0.08(\text { stat }) \pm 0.04(\text { syst })
\end{aligned}
$$

The fit to the invariant mass distribution, where both daughters are given a pion mass hypothesis, is shown in Fig. 2. The relevant yields extracted from the fit are shown in Tab. 1.

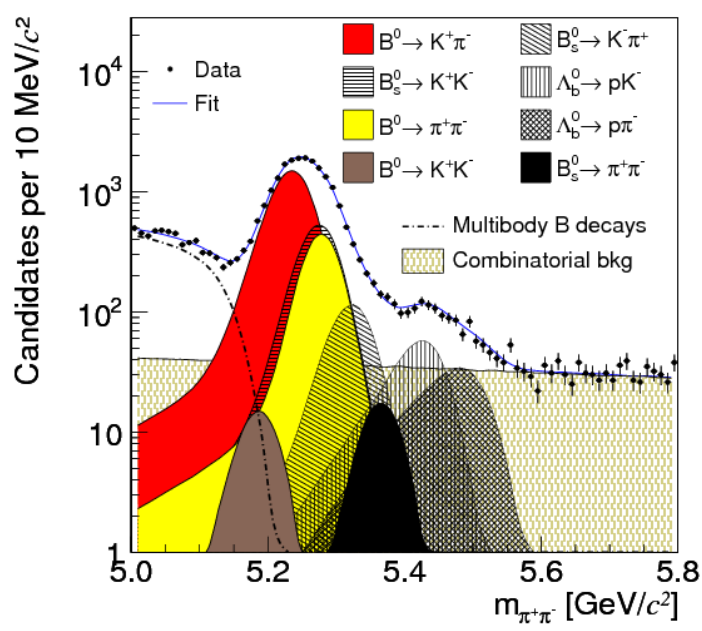

Figure 2: Fit to the $\pi^{+} \pi^{-}$invariant mass distribution of selected events collected at the CDF experiment.

Given the large number of $\Lambda_{b}^{0}$ baryons produced at the LHC, the LHCb collaboration will be able to increase the $\Lambda_{b}^{0} \rightarrow p \pi^{-}\left(K^{-}\right)$yields by a factor of 10 , yielding a precision of $\sim 3 \%$ on the measured value of CPV in $\Lambda_{b}^{0} \rightarrow p \pi^{-}\left(K^{-}\right)$decays.

\section{CP searches in $\Lambda_{b}^{0} \rightarrow \Lambda K^{+} K^{-}\left(\pi^{-}\right)$decays}

The charmless baryon decays $\Lambda_{b}^{0} \rightarrow \Lambda K^{+} K^{-}\left(\pi^{-}\right)$are of interest given that they occur via penguin processes and therefore will be suppressed in the SM and sensitive to interference effects 


\begin{tabular}{lcc}
\hline \hline Decay & $\mathcal{N}_{b \rightarrow f}$ & $\mathcal{N}_{\bar{b} \rightarrow \bar{f}}$ \\
\hline$B^{0} \rightarrow K^{+} \pi^{-}$ & $5313 \pm 109$ & $6348 \pm 117$ \\
$B_{s}^{0} \rightarrow K^{-} \pi^{+}$ & $560 \pm 51$ & $354 \pm 46$ \\
$\Lambda_{b}^{0} \rightarrow p \pi^{-}$ & $242 \pm 24$ & $206 \pm 23$ \\
$\Lambda_{b}^{0} \rightarrow p K^{-}$ & $271 \pm 30$ & $324 \pm 31$ \\
\hline \hline
\end{tabular}

Table 1: Yield taken from the fit to the $\pi^{+} \pi^{-}$invariant mass distribution.

from potential new physic particles. In addition, by using the decays $\Lambda_{b}^{0} \rightarrow \Lambda_{c}^{+}\left(\rightarrow \Lambda \pi^{+}\right) \pi^{-}$or $\Lambda_{b}^{0} \rightarrow \Lambda_{c}^{+}\left(\rightarrow \Lambda K^{+}\right) \pi^{-}$as control channels the production and reconstruction asymmetries can be largely cancelled. In Ref. [6] the LHCb collaboration observed for the first time the decays $\Lambda_{b}^{0} \rightarrow \Lambda K^{+} K^{-}\left(\pi^{-}\right)$. The CP asymmetries were measured to be

$$
\begin{gathered}
A_{C P}\left(\Lambda K^{+} \pi^{-}\right)=0.53 \pm 0.23 \pm 0.11 \\
A_{C P}\left(\Lambda K^{+} K^{-}\right)=0.28 \pm 0.10 \pm 0.07,
\end{gathered}
$$

which are less then $3 \sigma$ away from zero, indicating consistency with CP symmetry. The fits to the invariant mass distributions of events constructed under the $\Lambda K^{+} K^{-}$and $\Lambda K^{+} \pi^{-}$hypotheses are shown in Fig. 3.
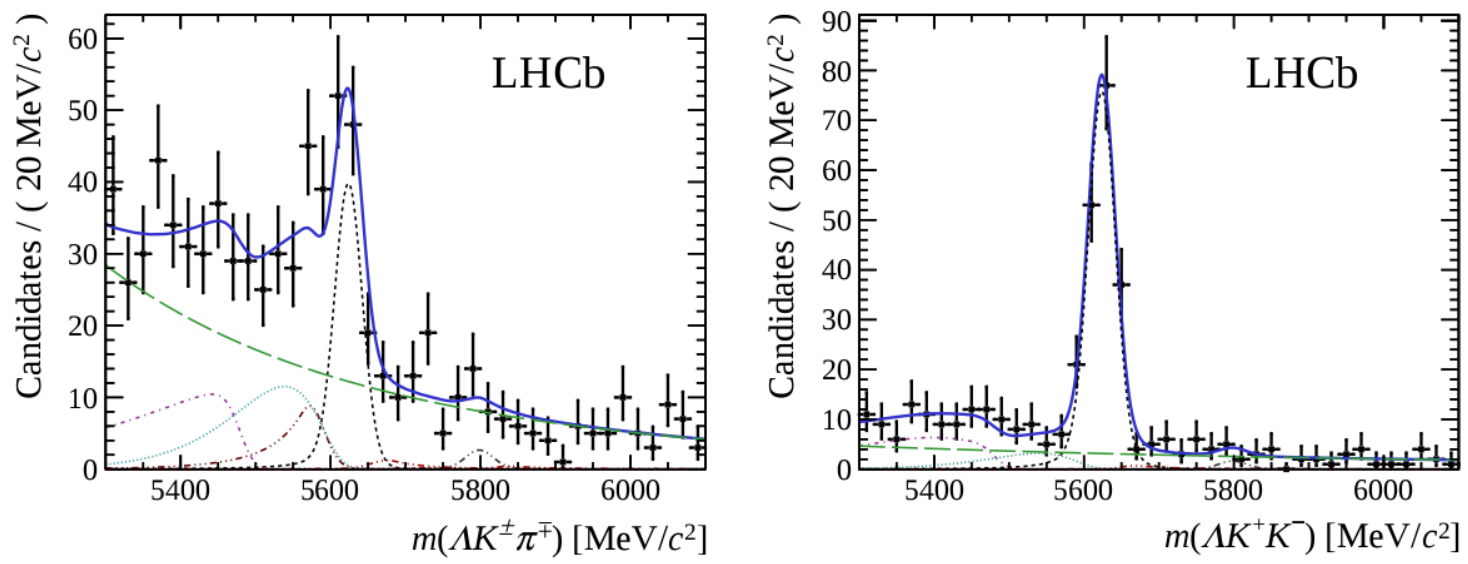

Figure 3: Fit to the $\Lambda K^{+} K^{-}$and $\Lambda K^{+} \pi^{-}$invariant mass distributions.

\section{First evidence for $\mathrm{CP}$ violation in baryons}

The decays $\Lambda_{b}^{0} \rightarrow p \pi^{-} \pi^{+} \pi^{-}$and $\Lambda_{b}^{0} \rightarrow p \pi^{-} K^{+} K^{-}$can occur either via a tree-level decay or via a penguin process. The Feynman diagram for both tree and loop-level processes can be seen in Fig. 4.

It is expected for the decays via tree-level diagrams and loop-level diagrams to have a similar amplitude, with tree-level processes being proportional to $V_{u b} V_{u d}^{*}$ and loop-level being proportional to $V_{t b} V_{t d}^{*}$. Again, the large relative phase between $V_{u b} V_{u d}^{*}$ and $V_{t b} V_{t d}^{*}$ makes this process particular sensitive to CPV effects. 

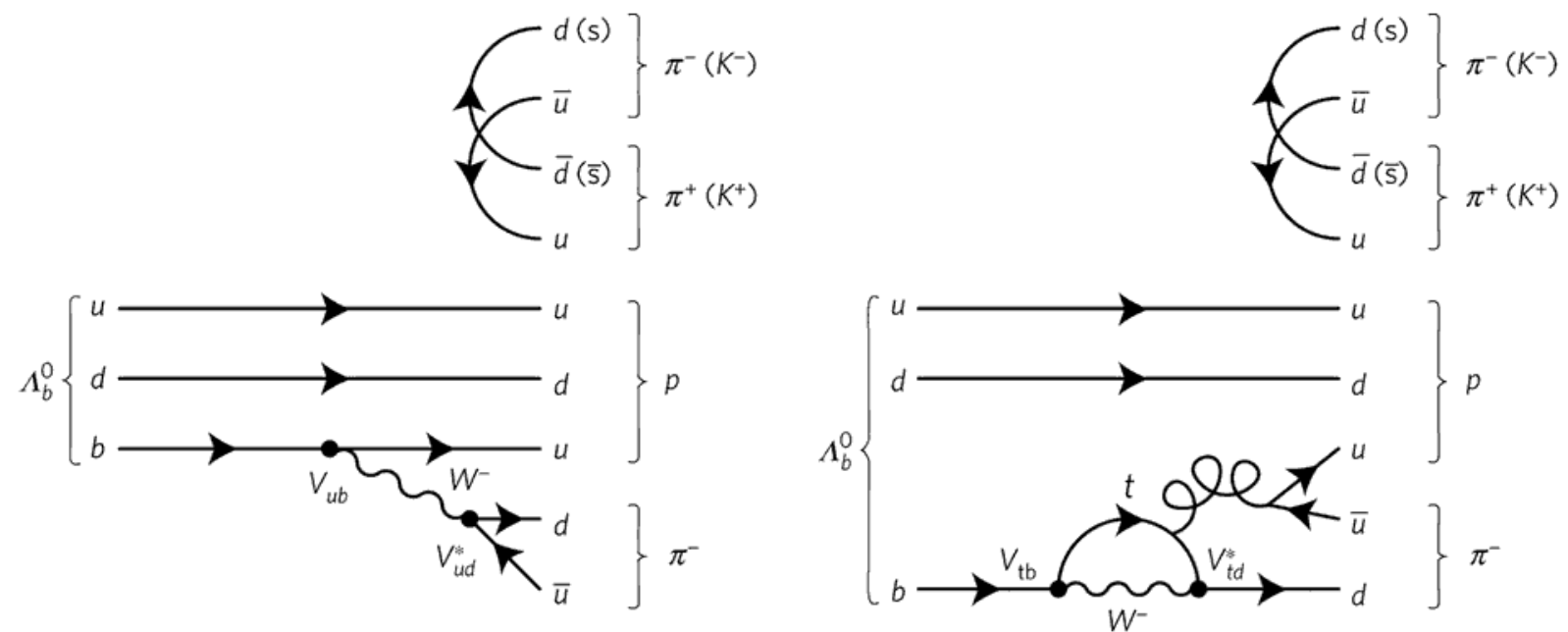

Figure 4: The tree and penguin diagrams for $\Lambda_{b}^{0} \rightarrow p \pi^{-} \pi^{+} \pi^{-}$and $\Lambda_{b}^{0} \rightarrow p \pi^{-} K^{+} K^{-}$decays.

The search for CPV in these decays is performed using triple-products, as introduced in section 2. Searches for CPV with triple-product asymmetries are particularly suited to charmless $\Lambda_{b}^{0}$ four-body decays to hadrons [7] thanks to their rich resonant substructure, particularly in the case of $\Lambda_{b}^{0} \rightarrow p \pi^{-} \pi^{+} \pi^{-}$.

The LHCb collaboration performed a search for CPV in $\Lambda_{b}^{0} \rightarrow p \pi^{-} \pi^{+} \pi^{-}$and $\Lambda_{b}^{0} \rightarrow p \pi^{-} K^{+} K^{-}$ decays in Ref. [8] using triple products suggested in Ref. [7] given as:

$$
\begin{aligned}
& \Lambda_{b}^{0}: C_{\hat{T}}=\mathbf{p}_{p} \cdot\left(\mathbf{p}_{h_{1}^{-}} \times \mathbf{p}_{h_{2}^{+}}\right) \propto \sin (\phi) \\
& \overline{\Lambda_{b}^{0}}: \bar{C}_{\hat{T}}=\mathbf{p}_{\bar{p}} \cdot\left(\mathbf{p}_{h_{1}^{+}} \times \mathbf{p}_{h_{2}^{-}}\right) \propto \sin (\bar{\phi}),
\end{aligned}
$$

where $h^{+}$is the pion (in the case of $\Lambda_{b}^{0} \rightarrow p \pi^{-} \pi^{+} \pi^{-}$) or kaon (in the case of $\Lambda_{b}^{0} \rightarrow p \pi^{-} K^{+} K^{-}$) with the same sign as the proton, and $h^{-}$is the pion with both the opposite sign to the proton and the greatest momentum in the rest frame of the $\Lambda_{b}^{0}$ (in the case of $\Lambda_{b}^{0} \rightarrow p \pi^{-} \pi^{+} \pi^{-}$) or the kaon with the opposite sign to the proton (in the case of $\Lambda_{b}^{0} \rightarrow p \pi^{-} K^{+} K^{-}$). The angle $\phi$ is shown in Fig. 5 and is defined as the angle between the two decay planes indicated in Fig. 5 in the rest frame of the $\Lambda_{b}^{0}$.

Asymmetries must than be constructed from the triple products, $C_{\hat{T}}$ and $\bar{C}_{\hat{T}}$, and these are defined as:

$$
\begin{aligned}
A_{\hat{T}} & =\frac{N_{C_{\hat{T}}}^{+}-N_{C_{\hat{T}}}^{-}}{N_{C_{\hat{T}}^{+}}^{+}+N_{C_{\hat{T}}}^{-}} \\
\bar{A}_{\hat{T}} & =\frac{\bar{N}_{-\bar{C}_{\hat{T}}}^{+}-\bar{N}_{-\bar{C}_{\hat{T}}}^{-}}{\bar{N}_{-\bar{C}_{\hat{T}}}^{+}+\bar{N}_{-\bar{C}_{\hat{T}}}^{-}},
\end{aligned}
$$

where $N_{C_{i}}^{+/-}$refers to the number of events with $C_{\hat{T}}$ greater or less than zero, respectively. 


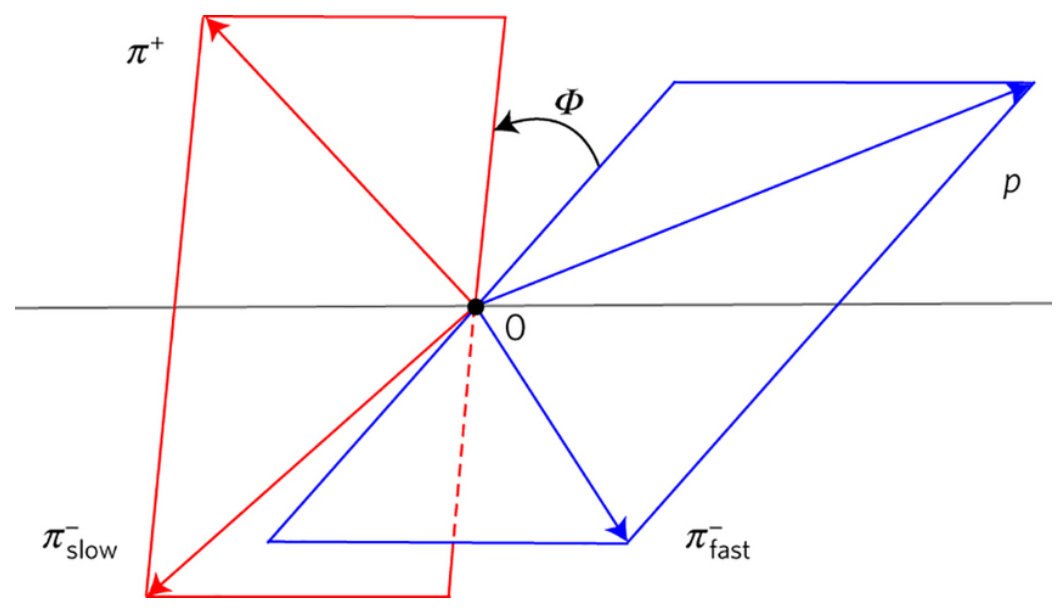

Figure 5: Schematic of the kinematics of the decay $\Lambda_{b}^{0} \rightarrow p \pi^{-} \pi^{+} \pi^{-}$used to define the triple-products.

From these asymmetries CP-odd observables can be constructed as:

$$
\begin{aligned}
& a_{P}^{\hat{T}-\text { odd }}=\frac{1}{2}\left(A_{\hat{T}}+\bar{A}_{\hat{T}}\right) \\
& a_{C P}^{\hat{T}-o d d}=\frac{1}{2}\left(A_{\hat{T}}-\bar{A}_{\hat{T}}\right),
\end{aligned}
$$

where a deviation from zero in $a_{P}^{\hat{T}-\text { odd }}\left(a_{C P}^{\hat{T}-\text { odd }}\right)$ would indicate $\mathrm{P}(\mathrm{CP})$ violation.

In four-body particle decays, the $\mathrm{CP}$ asymmetries may vary over the phase space due to resonant contributions and their interference effects. These effects may possibly cancel when integrated over the whole phase space. Thus for the $\Lambda_{b}^{0} \rightarrow p \pi^{-} \pi^{+} \pi^{-}$decays, the asymmetries are measured in different regions of phase space using two binning schemes (selected before looking at the data) as proposed in Ref. [9]. These two binning schemes are referred to as scheme A and scheme B. Scheme A involves splitting the phase space into bins depending on $\left\{m\left(p \pi^{+}\right), m\left(p \pi^{-}\right.\right.$slow $), m\left(\pi^{+} \pi^{-}\right.$slow $), m\left(\pi^{+} \pi^{-}\right.$fast $\left.),|\phi|\right\}$, with 12 bins being defined in total. Scheme B splits the phase space into bins depending on $|\phi|$.

The value of the CP-odd observables for the different binning schemes is shown in Fig. 6.

To calculate the deviation from the null hypothesis, a $\chi 2$ test is carried out for each observable across each binning scheme. Here, $\chi 2$ is defined as $R^{T} V^{-1} R$, where $R$ is the array of measurements and $V$ is the covariance matrix, which is the sum of the statistical and systematic covariance matrices. An average systematic uncertainty, which is dominated by experimental effects that could cause bias in the measured asymmetries, is assigned for all bins. The systematic uncertainties are assumed to be fully correlated and their contribution is small compared to the statistical uncertainties.

The resulting values for $a_{C P}^{\hat{T}-\text { odd }}$ are 2.0 and $3.4 \sigma$ for schemes A and B respectively. The resulting values for $a_{P}^{\hat{T}-o d d}$ are 2.8 and $2.4 \sigma$ for schemes A and B respectively. The combined significance for $a_{C P}^{\hat{T}-o d d}$ from the two schemes, where correlations between the two schemes are taken into account using pseudo experiments, is $3.3 \sigma$. The sensitivity of the result to the binning scheme used is tested by varying bin sizes and recalculating the global significances, which consistently remains above $3 \sigma$. 

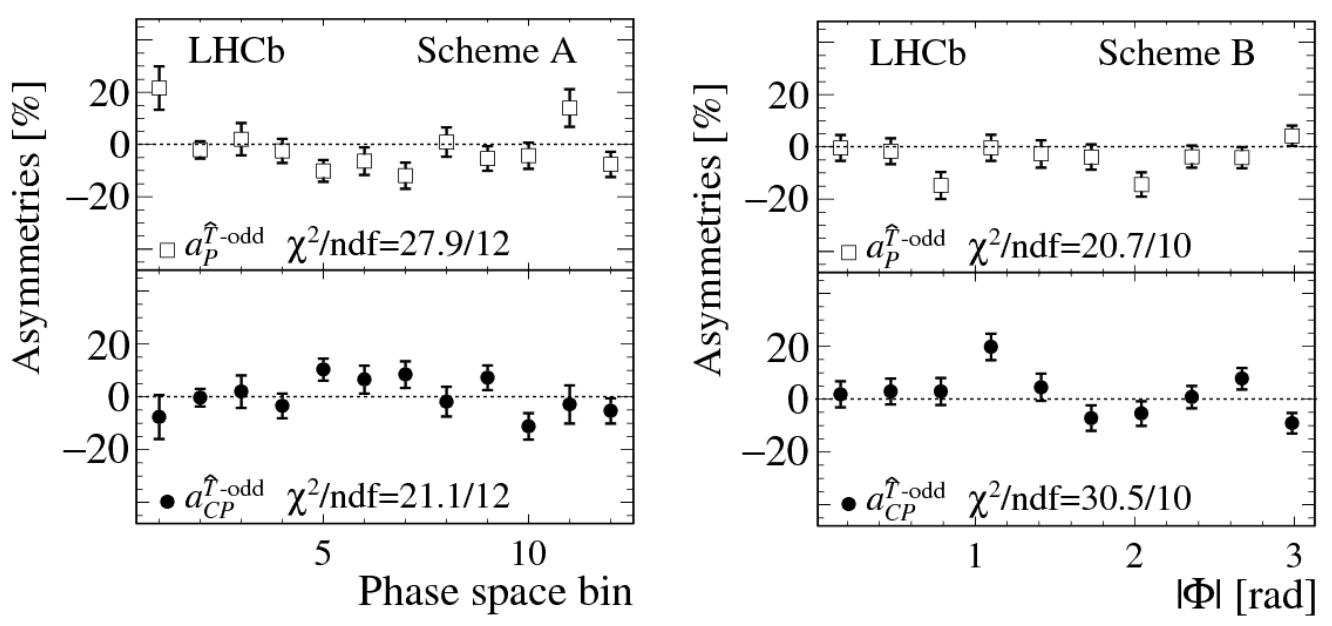

Figure 6: The value of the CP-odd observables, $a_{P}^{\hat{T}-o d d}, a_{C P}^{\hat{T}-o d d}$, for the different binning schemes are represented by open boxes and filled circles respectively. Scheme A shows the CP-odd observables as a function of bin number, where each bin number indicates a certain region in $\left\{m\left(p \pi^{+}\right), m\left(p \pi^{-}\right.\right.$slow $), m\left(\pi^{+} \pi^{-}\right.$slow $)$, $m\left(\pi^{+} \pi^{-}\right.$fast $\left.),|\phi|\right\}$ phase space.

For the decay $\Lambda_{b}^{0} \rightarrow p \pi^{-} K^{+} K^{-}$the lack of statistics means only two bins, dependent on the mass of the kaon-proton system, were used, and the results were consistent with P and CP symmetry.

This analysis constitutes the first evidence for CP-violation in the baryon sector.

\section{Searches for CPV in Flavour Changing Neutral Current (FCNC) processes}

\section{1 $\Lambda_{b}^{0} \rightarrow p K^{-} \mu^{+} \mu^{-}$decays}

The decay $\Lambda_{b}^{0} \rightarrow p K^{-} \mu^{+} \mu^{-}$occurs via a Flavour Changing Neutral Current (FCNC), as shown in Fig. 7, and therefore could be sensitive to new physics effects.
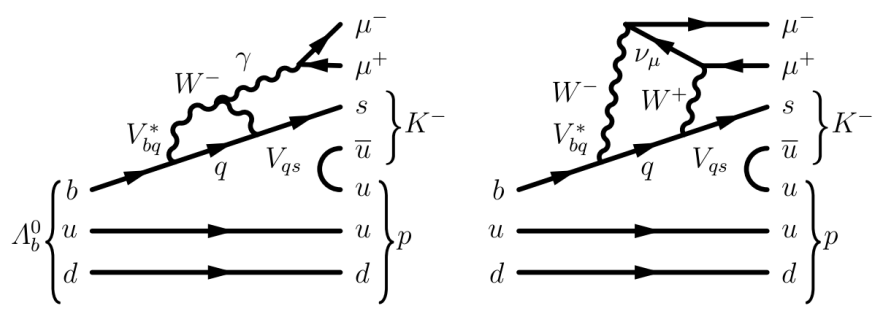

Figure 7: Possible Feynman diagrams from $\Lambda_{b}^{0} \rightarrow p K^{-} \mu^{+} \mu^{-}$decays.

Searches for CPV were made for this decay, both by using triple-products, similar to those outlined in Ref. 5 and by looking at $\Delta A_{C P}$, where the differential was taken with respect to the decay $\Lambda_{b}^{0} \rightarrow J / \psi p K^{-}$. The searches for CPV via either the triple products or $\Delta A_{C P}$ method are complementary, as the magnitude of CPV for each method is a function of the relative strong phase between the particle and anti-particle decay. Thus where one method maybe not be sensitive to CPV effects the other may still be. 
The fit to the invariant mass distribution for both the particle and anti-particle decay is shown in Fig. 8 and the resulting values for $\Delta A_{C P}$ and $a_{C P}^{\hat{T}-o d d}$ are measured as:
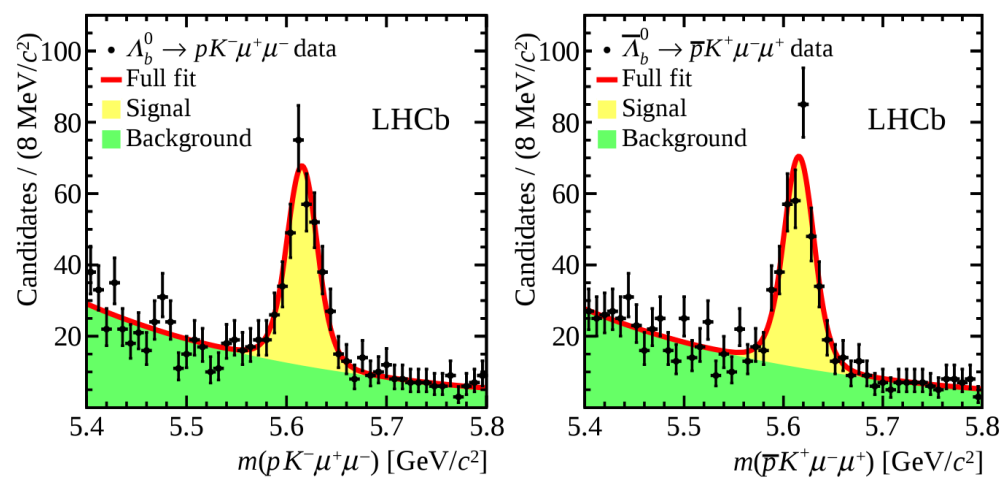

Figure 8: The fits to the invariant mass distribution of daughters reconstructed under the $p K^{-} \mu \mu\left(\bar{p} K^{+} \mu \mu\right)$ hypothesis.

$$
\begin{aligned}
\Delta A_{C P} & =(-3.5 \pm 5.0(\text { stat }) \pm 0.2(\text { syst })) \times 10^{-2} \\
a_{C P}^{\hat{T}-o d d} & =(1.2 \pm 5.0(\text { stat }) \pm 0.7(\text { syst })) \times 10^{-2},
\end{aligned}
$$

both of which are consistent with CP symmery [10].

\section{2 $\Lambda_{b}^{0} \rightarrow p \pi^{-} \mu^{+} \mu^{-}$decays}

The Cabibbo suppressed equivalent of the decay $\Lambda_{b}^{0} \rightarrow p K^{-} \mu^{+} \mu^{-}$is $\Lambda_{b}^{0} \rightarrow p \pi^{-} \mu^{+} \mu^{-}$, where the $s$ quark would be swapped for a $d$ quark in Fig. 7. This decay was observed by the LHCb collaboration in Ref. [11] and the fit to the invariant mass distribution is shown in Fig. 9. This decay channel has the potential to allow CPV searches to be carried out with the collection of more data during Run 2 of the LHC.

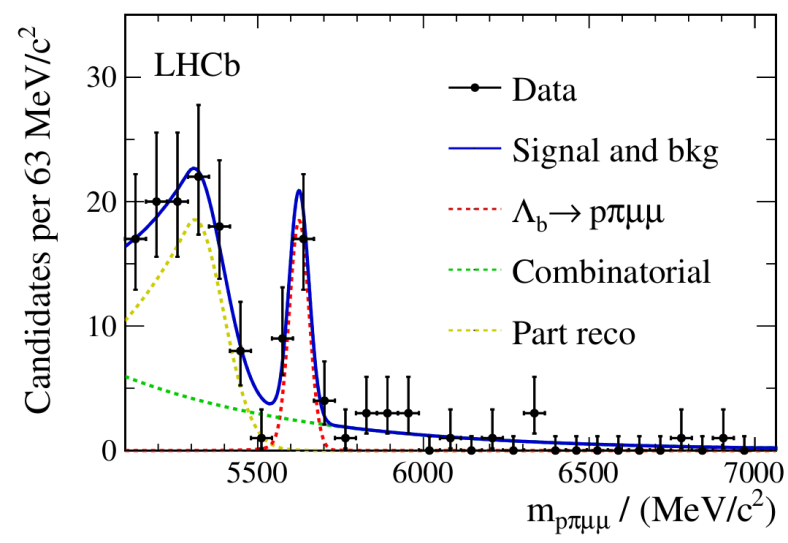

Figure 9: Fit to the invariant mass distribution of daughters reconstructed under the $p \pi \mu \mu$ mass hypothesis. 


\section{References}

[1] Christenson, J.H et al. Physical Review Letters 13 (1964), pp. 138-140

[2] Sachs, R. G. The Physics of Time Reversal (Univ. Chicago Press, 1987).

[3] Branco, G. C., Lavoura, L. and Silva, J. P. CP Violation (Oxford Univ. Press, 1999).

[4] Y. K. Hsiao and C. Q. Geng, Phys. Rev. D 91, no. 11, 116007 (2015) doi:10.1103/PhysRevD.91.116007 [arXiv:1412.1899 [hep-ph]].

[5] T. Aaltonen et al. (CDF Collaboration) Physical Review Letters 113 (2014), 242001

[6] R. Aaij et al. [LHCb Collaboration], JHEP 1605 (2016) 081 doi:10.1007/JHEP05(2016)081 [arXiv:1603.00413 [hep-ex]].

[7] M. Gronau and J. L. Rosner, Phys. Lett. B 749 (2015) 104 doi:10.1016/j.physletb.2015.07.060 [arXiv:1506.01346 [hep-ph]].

[8] R. Aaij et al. [LHCb Collaboration], Nature Phys. 13 (2017) 391 doi:10.1038/nphys4021 [arXiv:1609.05216 [hep-ex]].

[9] G. Durieux and Y. Grossman, Phys. Rev. D 92 (2015) no.7, 076013 doi:10.1103/PhysRevD.92.076013 [arXiv:1508.03054 [hep-ph]].

[10] R. Aaij et al. [LHCb Collaboration], JHEP 1706 (2017) 108 doi:10.1007/JHEP06(2017)108 [arXiv:1703.00256 [hep-ex]].

[11] R. Aaij et al. [LHCb Collaboration], JHEP 1704 (2017) 029 doi:10.1007/JHEP04(2017)029 [arXiv:1701.08705 [hep-ex]]. 